\title{
Improving on Nature: The Role of Nanomedicine in the Development of Clinical Natural Drugs
}

\author{
Authors \\ Anna Rita Bilia, Vieri Piazzini, Clizia Guccione, Laura Risaliti, \\ Martina Asprea, Giada Capecchi, Maria Camilla Bergonzi \\ Affiliation \\ Department of Chemistry, University of Florence, Sesto Fiorentino, \\ Florence, Italy \\ Key words \\ nanomedicine, nanoparticles, micelles and vesicles, artemisinin, \\ curcumin, salvianolic acid $B$, silymarin \\ $\begin{array}{ll}\text { received } & \text { September } 25,2016 \\ \text { revised } & \text { January } 16,2017\end{array}$ \\ accepted January 20, 2017 \\ Bibliography \\ DOI http://dx.doi.org/10.1055/s-0043-102949 \\ Published online February 8, 2017 | Planta Med 2017; 83: 366-381 \\ (c) Georg Thieme Verlag KG Stuttgart · New York | ISSN 0032-0943 \\ Correspondence \\ Prof. Dr. Anna Rita Bilia \\ Department of Chemistry, University of Florence \\ via Ugo Schiff 6, 50121 Sesto Fiorentino, Florence, Italy \\ Phone: + 390554573708 \\ ar.bilia@unifi.it
}

Eupporting information available online at

\begin{abstract}
Natural products have been used as a major source of drugs for millennia, and about half of the pharmaceuticals in use today are derived from natural products. However, their efficacy can be limited because of their low hydrophilicity and intrinsic dissolution rate(s), or physical/ chemical instability. In addition, they can present scarce absorption, poor pharmacokinetics and bioavailability, scarce biodistribution, first-pass metabolism, trivial penetration and accumulation in the organs of the body, or low targeting efficacy. Novel nanoformulations based on drug delivery systems, namely nanoparticles, micelles, and vesicles, offer significant promise in overcoming these limitations. Nowadays, nanomedicine is crucial in developing appropriate therapeutic treatments of essential drugs, specifically antitumor and antiparasistic agents (i.e., Taxol, vincristine, camptothecin, doxorubicin, artemisinin) and other emerging molecules with pleiotropic functions (i.e., resveratrol, curcumin, salvianolic acid B, honokiol). Additionally, the number of nanoformulations developed with flavonoids, in particular rutin, quercetin, silymarin, and green tea catechins, is constantly increasing, and a significant number of publications have appeared in the last decade pertaining to nanoformulations based on extracts and essential oils. Most of these studies report very promising nanoformulations with sustained release and improved bioavailability at much lower doses than conventional preparations, and in many cases, also a better safety profile.
\end{abstract}

\section{Historical Overview of the Role of Natural Products in Medicine}

Natural products from plants, animals, and minerals have played a key role in treating and preventing human diseases since ancient times and they still represent a significant source of modern drugs. Currently, the global market of natural products, which is mainly derived from the plant kingdom, is estimated at more than US\$80 billion and it continues to grow. Herbal drugs and extracts sold as dietary supplements, food, or herbal medicinal products are the principal products, while single isolated constituents represent a lesser proportion. In addition, according to the WHO, between 65 and $80 \%$ of populations in developing countries presently use medicinal plants as therapeutic remedies [1].

Indeed, natural products still represent a main source of drugs thanks to their enormous structural and chemical diversity to which synthetic libraries cannot compare [2].

Over time, drug discovery has undergone many transformations, and in the last decade, it has become clear that the use of drugs targeting single sites have low therapeutic value against multifactorial and complex diseases, especially cancer and diabetes [3]. By contrast, diverse natural products can modulate multiple targets activating various signalling or functional pathways [4].

The tangible importance of natural products to the drug discovery process and their possible role in therapy is unquestionable. Conversely, their efficacy is frequently scarce because of low hydrophilicity and an intrinsic dissolution rate, and/or physical or chemical instability. In addition, they can have low absorption, scarce biodistribution, first-pass metabolism, poor penetration and accumulation in the organs of the body, or trivial targeting efficacy. Without a doubt, extracts have a generally better therapeutic performance than single constituents. Occasionally, the constituents provide synergistic or simply additive action resulting in an enhanced therapeutic value. Conversely, extracts are usually very complex mixtures, made up of molecules with different solubility and chemical structures. They are generally considered poor drug candidates because they commonly need repeated administrations or higher doses with respect to the single 


\begin{tabular}{|c|c|}
\hline \multicolumn{2}{|c|}{ ABBREVIATIONS } \\
\hline $\mathrm{A}-\mathrm{CL}$ & artemisinin-loaded conventional liposomes \\
\hline A-PL & artemisinin-loaded PEGylated liposomes \\
\hline $\mathrm{AC}-\mathrm{CL}$ & $\begin{array}{l}\text { artemisinin-curcumin-loaded conventional } \\
\text { liposomes }\end{array}$ \\
\hline AC-PL & $\begin{array}{l}\text { artemisinin-curcumin-loaded pegylated } \\
\text { liposomes }\end{array}$ \\
\hline AIDS & acquired immune deficiency syndrome \\
\hline ALT & alanine aminotransferase \\
\hline AP & alkaline phosphatase \\
\hline ART & artemisinin \\
\hline ART-L & artemisinin loaded in long circulating liposomes \\
\hline ART-LTf & $\begin{array}{l}\text { artemisinin loaded in long circulating liposomes } \\
\text { actively targeted with transferrin }\end{array}$ \\
\hline ASGR & asialoglycoprotein receptor \\
\hline AST & serum aspartate aminotransferase \\
\hline AUC & area under the plasma concentration-time curve \\
\hline \multicolumn{2}{|r|}{ under the curve from time $0 \mathrm{tc}$} \\
\hline BCS & biopharmaceutics classification system \\
\hline CD & cyclodextrin \\
\hline DHA & dihydroartemisinin \\
\hline EMA & European Medicines Agency \\
\hline EPR & enhance permeation and retention effect \\
\hline FDA & Food and Drug Administration \\
\hline Fol & folate \\
\hline GRAS & generally recognized as safe \\
\hline $\mathrm{HP}-\beta-\mathrm{CD}$ & hydroxypropyl- $\beta$-cyclodextrin \\
\hline HSV & herpes simplex virus \\
\hline i. p. & intraperitoneal \\
\hline MDR & multidrug resistance \\
\hline miRNA & microribonucleic acid \\
\hline NLCs & nanostructured lipid carriers \\
\hline PEG & polyethylene glycol \\
\hline PLA & polylactic acid \\
\hline PLGA & polylactic glycolic acid \\
\hline RES & reticuloendothelial system \\
\hline SLNs & solid lipid nanoparticles \\
\hline $\mathbf{t}(1 / 2 \beta)$ & elimination half-life \\
\hline TEM & transmission electron microscope \\
\hline$T_{\max }$ & time at which the $C_{\max }$ is observed \\
\hline TOPO & topoisomerase \\
\hline WHO & World Health Organization \\
\hline
\end{tabular}

comes from the ancient Greek võvos through the Latin nanus meaning literally "dwarf" and, by extension, "very small”, namely between 50 and $300 \mathrm{~nm}$. Numerous nanosized drug delivery systems have already entered into clinical use. The most pressing challenge for the near future is the design of multifunctional, structured materials able to target specific tissues or organs or containing functionalities to allow transport across biological barriers. These delivery systems are smartly designed to tag a variety of chemical, molecular, and biological entities. A successful drug carrier system needs to demonstrate optimal drug loading and release properties, a long shelf life, and exert a much higher therapeutic efficacy as well as lower side effects [7].

The promising therapeutic activities of many isolated natural products or extracts have encouraged nanotechnologists to design and formulate useful nanoformulations to improve solubility, stability, cellular uptake/internalization efficacy, specificity, tolerability, and therapeutic index. Currently, nanotechnology has an enormous impact in medical technology, significantly improving the performance of drugs in terms of efficacy, safety, and patient compliance. Some paradigmatic success stories concerning both single constituents and refined/native extracts of nanoengineered formulations are reported in this review.

\section{Nanomedicine: Merely a Question of Size?}

Bioavailability is defined by the EMA as "the rate and extent to which the active ingredient or active moiety is absorbed from a drug product and becomes available at the site of action". Bioavailability is usually assessed by determining the AUC (AUC-time relationship after administration of a hypothetical drug.). After intravenous administration, it is assumed that the given dose of the drug is $100 \%$ bioavailable, since the drug is introduced directly into the systemic circulation. All other forms of systemic administrations, including oral, intramuscular, subcutaneous, etc., generally present a bioavailability of less than $100 \%$ [8]. In particular, after oral administration, the solubility, dissolution rate, gastrointestinal permeability, first-pass metabolism, and susceptibility to efflux mechanisms are fundamental parameters that control rate and extent of drug absorption and its bioavailability. The BCS is a scientific classification of a drug substance based on its aqueous solubility and intestinal permeability that correlates in vitro dissolution and in vivo bioavailability of drug products ( $\bullet$ Fig. 1 ).

The BCS takes into account two major factors: solubility and intestinal permeability, which governs the rate and extent of oral drug absorption from solid dosage forms and, ultimately, their bioavailability. For this reason, BCS is a fundamental tool in drug development, especially in the development of oral drug formulations. A drug is considered highly soluble when the highest strength is soluble in $250 \mathrm{~mL}$ (this volume is derived from typical bioequivalence study protocols) or less of aqueous media over the $\mathrm{pH}$ range of 1.0-7.5; otherwise, the drug substance is considered poorly soluble. Permeability classification is based directly on the extent of intestinal absorption of a drug substance in humans or indirectly on the measurements of the mass transfer rate across the intestinal membrane. A drug substance is considered highly permeable when the extent of intestinal absorption is $90 \%$ or 
higher based on mass balance or in comparison to an intravenous reference dose. BCS class I drugs are those molecules having high solubility and high permeability and are considered prototypical drugs. BCS class II drugs have high permeability and studies are focused on their solubility enhancement. Bioavailability of class III drugs is permeability rate limited, but dissolution is likely to occur rapidly. In case of BCS class IV, bioavailability is limited by both dissolution as well as intestinal permeability, and they may suffer from an inadequate, or highly variable, rate and/or extent of drug absorption (sometimes as a function of food in the stomach, i.e., fed/fasted variability) [9]. Increased drug water solubility and permeability could be achieved via different strategies, including the chemical approach to obtain more bioavailable semisynthetic compounds or prodrugs and the technological approach in order to develop proper drug delivery systems $[10,11]$. A simple and affordable technique to enhance solubility of a drug is to modify its physical characteristics by reducing particle size and/or modifying its crystal habit. Apart from conventional micronizing techniques, particle technology now deals with various nanoparticle engineering processes as promising methods to improve drug solubility. Nanopowders in the solid state (either amorphous or crystalline) have a typical size range of $10-1000 \mathrm{~nm}$. Nanoscale materials have far greater surface areas than similar masses of larger scale materials, resulting in an increased dissolution. To quantify the potential benefit of a nanosized powder to hugely improve their solubility, the following principles can be applied. A solid cube of a material measuring $1 \mathrm{~mm}$ per side (the typical size of conventional drugs) has $6 \mathrm{~mm}^{2}$ of surface area. When the size is reduced to $10 \mu \mathrm{m}$, the total surface area is $600 \mathrm{~mm}^{2}$, which becomes $60000 \mathrm{~mm}^{2}$ if the cube is $100 \mathrm{~nm}$ per side ( $\mathbf{F i g} .2$ ). Only a minimum amount of surfactants or hydrophilic polymers is added to the formulation for steric and electrostatic surface stabilization. They form a thin coating that inhibits the formation of cakes or simply the aggregation of the crystals/amorphous powders, which can severely affect the dissolution behavior. Nanopowders, both in the form of nanocrystals and amorphous systems, can, at least theoretically, improve all common drug administration routes (oral, parenteral, transdermal, transmucosal, ocular, pulmonary), controlling the rate and extent of drug absorption and, ultimately, its bioavailability $[12,13]$.

Indeed, the formulation of "nanosized" vectors offers additional advantages that are not achievable with simple nanopowders. In addition to the increased solubility (typical of nanopowders), these carriers can enhance photo- and chemical stability, bioavailability, and, as a consequence, efficacy. Nanovectors are also capable of addressing several drug delivery problems, i.e., overcoming multidrug-resistance phenomena, penetrating cellular barriers that may limit the intended target site, and improving in vivo efficacy of the drug $[14,15]$. A nanotechnology approach could be attractive in modulating both drug pharmacokinetics and biodistribution, thus decreasing potential side effects by leaving the normal sensitive cells unharmed. Additionally, nanovectors could mask unpleasant taste or limit the volatility of a molecule/extract (i.e., essential oil) $[16,17]$.

Size, shape, charge, hydrophobic/hydrophilic properties, and surface chemistry play astonishing roles in the ultimate success of nanocarriers' optimizing properties, namely, reactivity,

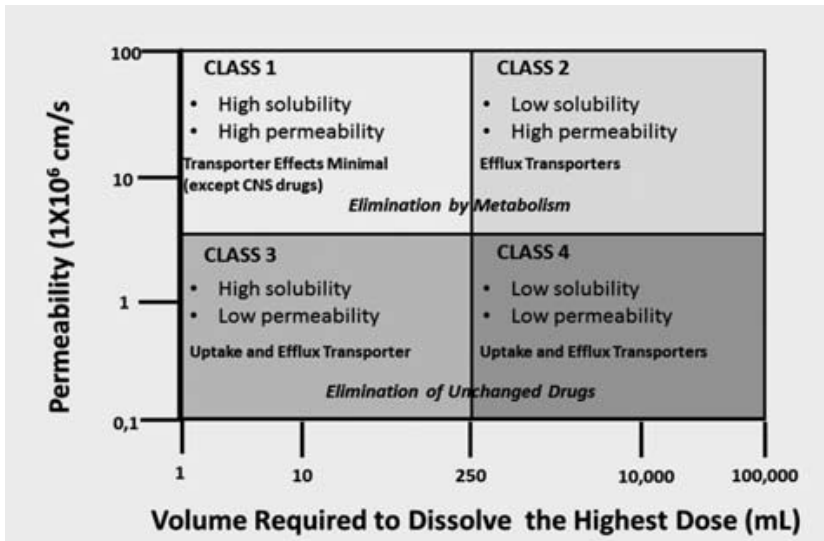

- Fig. 1 BCS of drug products.

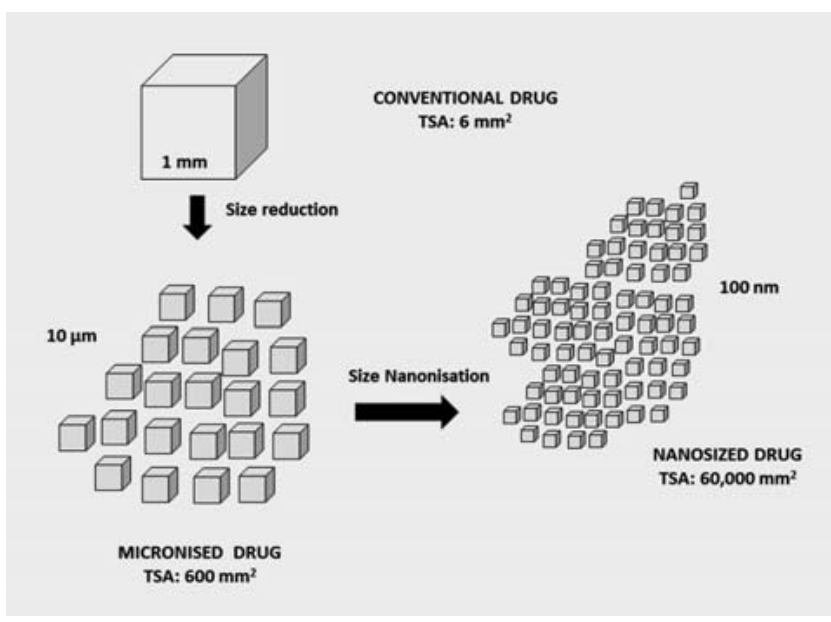

Fig. 2 Total surface areas of conventional, micronized, and nanosized drug powders.

strength, electrical characteristics, and in vivo behavior, including active intracellular delivery and improved pharmacokinetic and pharmacodynamics profiles of drugs. In this way, some physiological parameters including hepatic filtration, tissue extravasation, tissue diffusion, and kidney excretion may be modified. A schematic representation of these factors is reported in $\mathbf{F}$ Fig. 3 .

The size of nanosystems is directly related to the cellular uptake rate (the best is $200-300 \mathrm{~nm}$ in diameter) and to the time they remain in blood circulation (those having a diameter less than $10 \mathrm{~nm}$ are cleared via glomerular filtration in the kidneys) [18].

Surface modification of nanosystems can be achieved by coating the exterior with mucoadhesive molecules (i.e., chitosan), enhancing drug absorption, or using hydrophilic polymers, for example, PEG, which can limit opsonization by the cells of the RES. The nanocarriers that escape RES can circulate in the bloodstream for longer periods and have a greater chance of reaching the targeted tissues. It has been observed that under certain circumstances, namely, inflammation/hypoxia, tumors, and infarct, the 


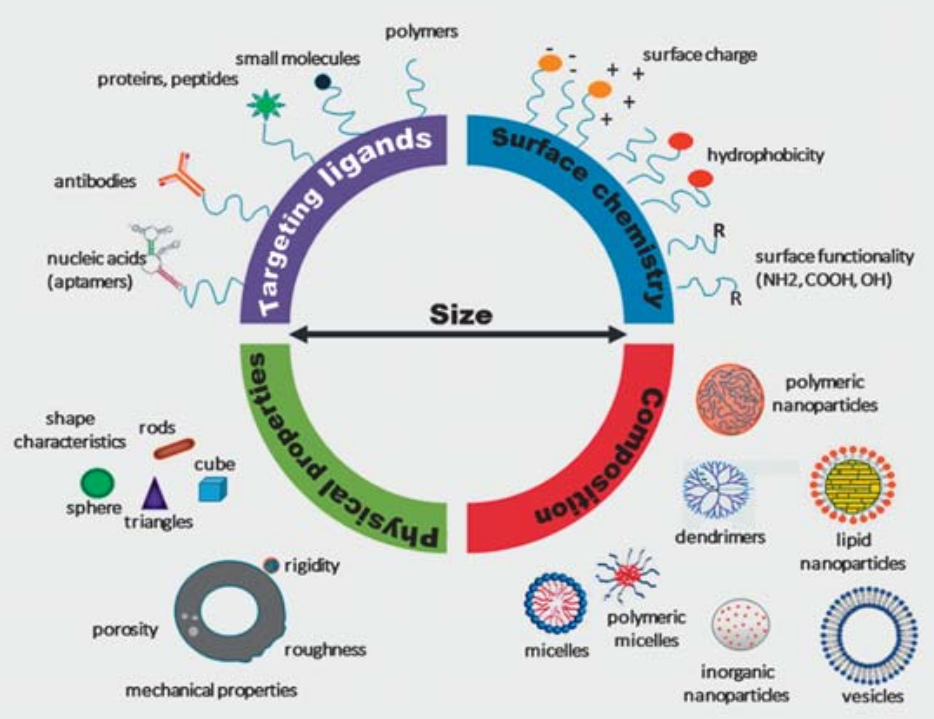

- Fig. 3 Roles of size, shape, charge, hydrophobic/hydrophilic character, and surface chemistry of nanocarriers to optimize in vivo behavior including active intracellular delivery and improved pharmacokinetics and pharmacodynamics of drugs, modifying some physiological parameters, including hepatic filtration, tissue extravasation, tissue diffusion, and kidney excretion.

- Fig. 4 The role of EPR effects for nanocarrier behavior in healthy tissues (a), their accumulation in inflamed or tumor tissues (b), and active drug targeting of nanocarriers (c).

endothelial lining of the blood vessel wall becomes more permeable than in the physiological state of the tissue. Nanocarriers can better penetrate tumors or body inflamed regions, due to their leaky constitution and pores ranging from 100-1000 nm in diameter ( $\bullet$ Fig. 4).

An alternative strategy of passive targeting through the EPR effect is decoration of the nanocarriers surface with targeting elements to maximize their accumulation at the site of interest, de- fined as active drug targeting ( $\bullet$ Fig. 4). This approach represents an ideal therapy to treat a disease conserving normal tissues and cells. A large body of evidence suggests that these targeting strategies could overcome drug resistance and side effects to the vital organs. Diverse targeting elements can be used to decorate the surface of the nanocarriers, namely, antibodies, small molecules, proteins, and peptides ( $\triangleright$ Fig. 3 ) [19]. 
As a consequence, nanocarriers can enhance delivery to target tissue because of the increased solubility and permeability of the drug $[20,21]$, thereby reducing the dosing frequency and improving patient compliance $[20,22,23]$. These nanosystems can also minimize side effects [24] and sustain drug release over a prolonged period. Nanovectors are generally suitable for parental, oral, mucosal, pulmonary, ocular, dermal, and transdermal delivery. Opportunely designed, they are able to cross physiological barriers, i.e., blood-brain barrier, blood-cerebrospinal fluid barrier, blood-retinal barrier, and the other barriers of the eye (corneal, tear film, and aqueous) ( $\triangleright$ Fig. 5).

There are four distinct mechanisms for molecules to cross the mucosal membranes: via the paracellular and transcellular routes, and carrier-mediated and receptor-mediated transport ( $\bullet$ Fig. 6 ). Absorption through each pathway is dependent on different physical characteristics, including molecular weight, hydrophobicity, ionization constants, and stability of molecules as well as biological barriers. Some properties, i.e. mucoadhesion and retention by the mucosa, are strongly desired. In addition, the ability to penetrate through mucus and reach an adherent mucus layer or epithelium surfaces to prolong extracellular drug release, or facilitate mucosal permeation and intracellular drug delivery are also welcome.

Topical administration of nanocarriers produces surface effects (film formation and occlusive effects), local effects (drug delivery in the epidermis and dermis), and systemic effects (deeper drug permeation and transdermal delivery resulting in regional or systemic effects). The skin is the largest organ of the body, accounting for more than $10 \%$ of body mass, enabling a more intimate interaction with the environment. Essentially, it consists of four layers, the stratum corneous is the outer layer of the skin (nonviable epidermis), and forms the rate-controlling barrier for diffusion for almost all compounds. It is composed of dead, flattened, keratin-rich cells, the corneocytes. A complex mixture of intercellular lipids, namely, ceramides, free fatty acids, cholesterol, and cholesterol sulphate, surrounds these dense cells. The remaining three layers are the epidermis (viable epidermis), the dermis, and the subcutaneous tissue. There are also several associated appendages, hair follicles and sweat glands, which form shunt pathways through the intact epidermis; they occupy only $0.1 \%$ of the total human skin ( $\vee$ Fig. 7). Permeation of drugs through the skin includes diffusion through the intact epidermis and through the skin appendages. Drug permeation through the skin is usually limited by the stratum corneum. Two pathways through the intact barrier may be identified, the intercellular and transcellular routes as shown in - Fig. 7. The intercellular lipid route is between the corneocytes. Lipids in the skin barrier are crucially important for transepidermal diffusion of the lipid and amphiphilic molecules, occupying those spaces for the insertion and migration through intercellular lipid layers of such molecules. The predominant diffusion of hydrophilic molecules is "laterally" along surfaces of the less abundant, water-filled interlamellar spaces. The transcellular route includes the crossing through the corneocytes and the lipidic phase. Intracellular macromolecular matrix within the stratum corneum abounds in keratin, which does not contribute directly to the skin diffusive barrier but it supports mechanical stability and thus intactness of the stratum corneum. Recently, follicular pene-

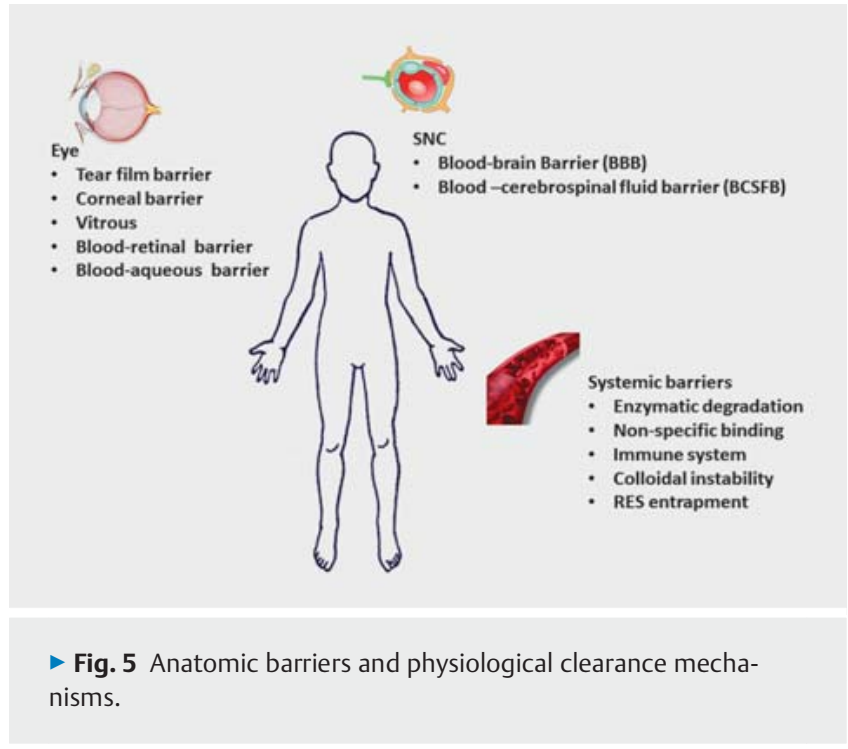

tration has become a focus of interest in the treatment of skin diseases. However, due to the fact that follicular orifices occupy only a very small portion of the total skin surface area, studies are frequently inconsistent [25-27].

\section{Paradigmatic Examples of Nanovectors Loaded with Natural Products to Optimized Therapeutic Activity}

Different types of nanomaterials can be used to prepare nanocarriers capable of being loaded with hydrophobic and hydrophilic drugs. Since the human body contains mostly water, the ability to successfully deliver hydrophobic drugs is a major therapeutic benefit of nanocarriers. Some of these, i.e., vesicles, are able to contain either hydrophilic or hydrophobic drugs depending on the orientation of the amphiphilic molecules. Generally, nanocarriers are classified as polymer-based systems and lipid-based systems ( $\triangleright$ Fig. 8).

Polymeric nanocarriers are composed of natural (proteins and polysaccharides), semisynthetic or synthetic polymers, generally represented by nanospheres or nanocapsules. Nanospheres are matrix systems in which the drug is physically and uniformly dispersed, while nanocapsules are the system in which the drug is confined to a cavity surrounded by a polymeric membrane ( $\bullet$ Fig. 8).

Polymeric micelles are made up of block copolymers that consist of hydrophobic and hydrophilic monomer units dispersed in an aqueous media. Two monomers with different hydrophobicity can be conjugated to form a core-shell micelle structure, where the hydrophilic and hydrophobic blocks form the micelle shell and core, respectively. Common polymers used to prepare micelles are Pluronics (polyoxyethylene polyoxypropylene block copolymers) that spontaneously self-associate in aqueous solution ( $\triangleright$ Fig. 8). 


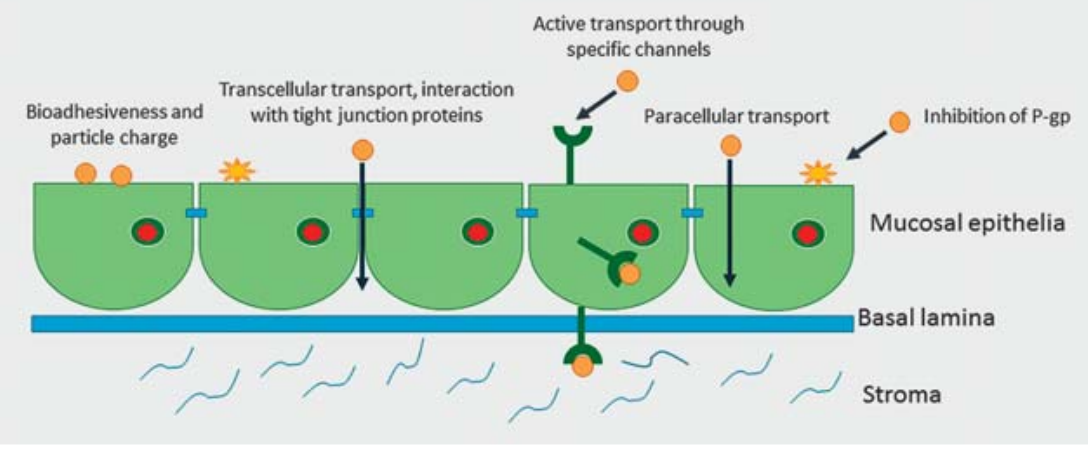

- Fig. 6 Mechanisms of enhanced absorption of nanoparticles through mucosal epithelia.

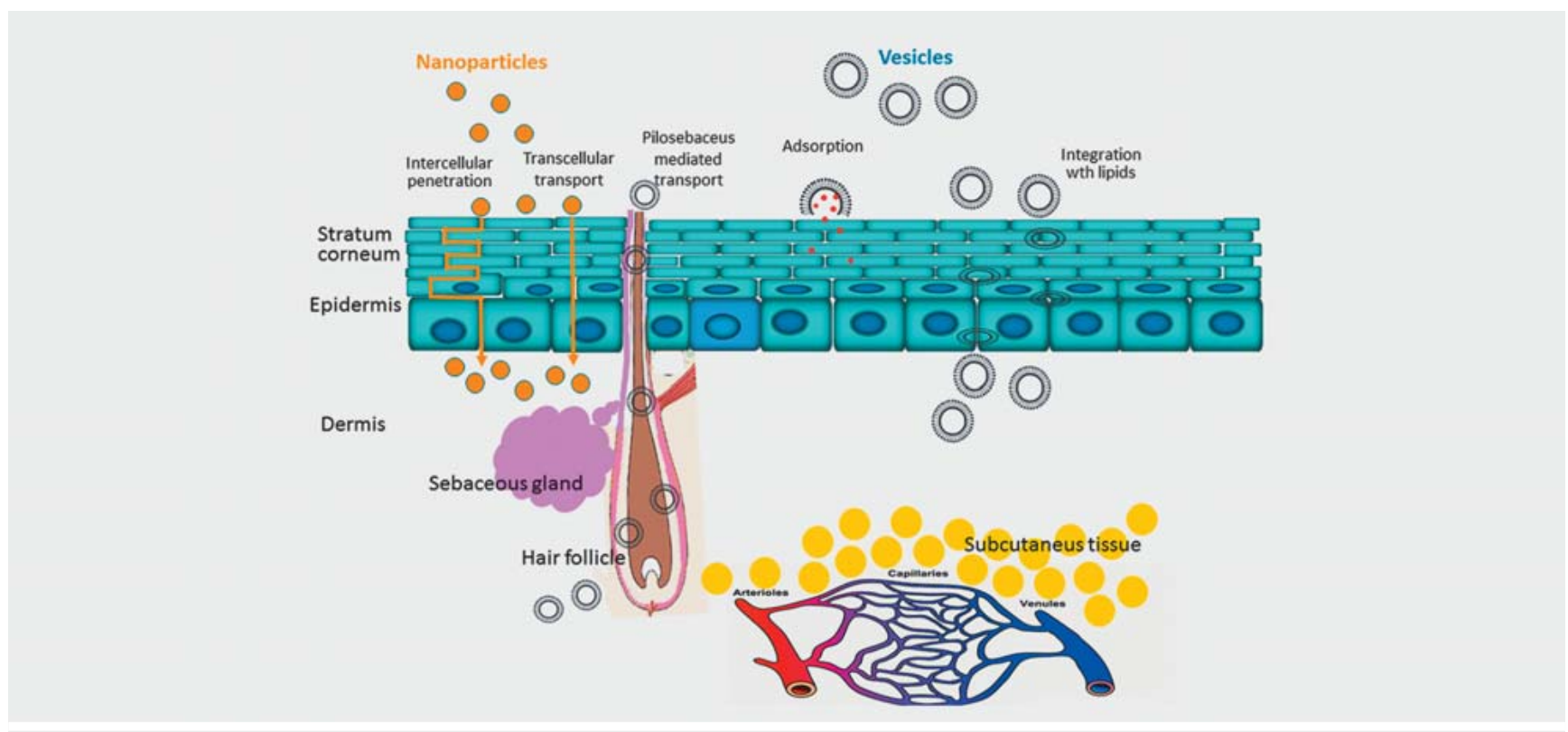

- Fig. 7 Permeation mechanisms of nanocarriers in the skin.

Lipid-based nanocarriers include nanometric-scaled emulsions, namely, micro- and nanoemulsions, vesicles including liposomes and niosomes, micelles, and nanoparticles divided roughly into SLNs and NLCs ( $\bullet$ Fig. 8). Vesicles and micelles are aggregates of amphiphilic lipids that organize themselves spontaneously. Vesicles are characterized by bilayer structures that are suitable to load both hydrophilic and hydrophobic compounds. Micelles can only load lipophilic molecules in the lipid core. SLNs and NLCs are solid particles at room temperature and human body temperatures. They present a lipid core, which makes these carriers suitable for entrapment of lipophilic compounds. Lipids and surfactant agents are generally selected from a plethora of edible constituents and GRAS approved compounds.

\section{Paclitaxel (Taxol) nanocarriers: improved water solubility, efficacy and safety profiles}

Paclitaxel (Taxol), the most exciting plant-derived anticancer drug discovered in recent years, promotes the assembly of tubulin into microtubules. It was first isolated from the bark of the Pacific yew (Taxus brevifolia L.). It was approved for clinical use against ovarian cancer in 1992 and against breast cancer in 1994. Nowadays, the drug represents a first-line treatment for ovarian, breast, lung, and colon cancer and a second-line treatment for AIDS-related Kaposi's sarcoma. It is so effective that some oncologists refer to the period before 1994 as the "pre-Taxol" era for treating breast cancer. Taxol is typically administered intravenously, but due to the low water solubility, the i.v. route of administration needs polyethoxylated castor oil (Cremophor EL, a non-ionic surfactant) and ethanol (50:50) as cosolvents. Consequently, this formulation is associated with serious and dose-limiting toxicities. Cremophor EL itself causes hypersensitivity reactions (premedication 


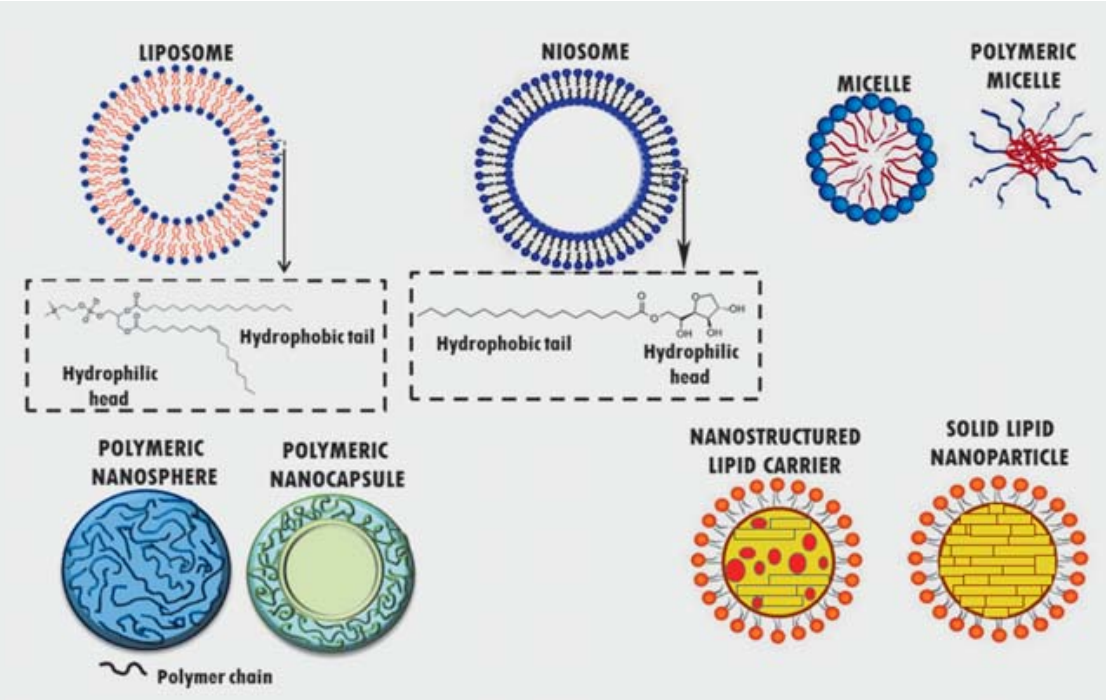

- Fig. 8 Structures of the diverse nanocarriers.

with oral dexamethasone and diphenhydramine are required) and it is known to leach out plasticizers from standard intravenous injection tubing, necessitating special infusion sets. Many studies have been developed in the last 15 years in an attempt to overcome these issues. A number of novel paclitaxel nanoformulations investigated in clinical trials are now available. They include polymeric nanoparticles, lipid-based formulations, polymer conjugates, inorganic nanoparticles, nanocrystals, and CD nanoparticles. Ping and Mumper [28] reported a comprehensive review in 2013.

The clinically approved albumin nanoparticle formulation of paclitaxel, Abraxane, in the form of nanospheres (130 nm in diameter) has dramatically increased the bioavailability of paclitaxel resulting in higher intratumor concentrations facilitated by albumin receptor (gp60)-mediated endothelial transcytosis [29].

Paclitaxel loaded in these nanoparticles can be reconstituted in normal saline solution at concentrations of $2-10 \mathrm{mg} / \mathrm{mL}$, compared with $0.3-1.2 \mathrm{mg} / \mathrm{mL}$ used for paclitaxel. Paclitaxel loaded in albumin nanoparticles undergoes biphasic elimination (twocompartment model of disposition) with a terminal half-life of $27 \mathrm{~h}$ ( $5.8 \mathrm{~h}$ for paclitaxel solution). The clearance is $43 \%$ slower $\left(15 \mathrm{~L} / \mathrm{h} / \mathrm{m}^{2}\right)$ and the mean volume of distribution is $632 \mathrm{~L} / \mathrm{m}^{2}$ (indicating extensive extravascular distribution) when compared with the paclitaxel solution. The drug exposure (AUC) was proportional to the dose in the range of $80-375 \mathrm{mg} / \mathrm{m}^{2}$. The nanoformulation does not require premedication, with a considerably shorter recommended infusion time (i.e., 30 min vs. $2-4 \mathrm{~h}$ for the paclitaxel solution) [30].

Accordingly, this nanoformulation represents a very successful drug delivery system of paclitaxel, approved in different countries for the treatment of metastatic breast cancer. Since 2012 it represents a first-line treatment for advanced non-small cell lung cancer in combination with carboplatin for patients who are not candidates for curative surgery or radiation therapy. Abraxane has also shown aptitude for treatment of advanced pancreatic cancer and the US FDA has expanded approval to include advanced pancreatic cancer in 2013. Abraxane has been associated with fewer acute adverse drug reactions and significant reductions in health care resources, physician/nurse time, and overall drug delivery costs compared to conventional Taxol formulations [31, 32].

\section{Other anticancer natural drugs}

Anthracyclines (doxorubicin, daunorubicin, and idarubicin) are among the most effective and commonly used chemotherapeutic drugs. The mechanisms of antitumor activity of anthracyclines are well documented, wherein anthracyclines are able to diffuse across the cell membrane, intercalate between DNA base pairs, target TOPO II, and induce cell apoptosis. Unfortunately, the clinical use of anthracyclines has been limited by their severe cardiotoxicity and the development of multiple drug resistance. In general, many patients achieve a complete remission when initially treated with anthracyclines; however, ca. $70 \%$ of the patients ultimately experience a relapse of the disease, and the treatment failure is mainly due to multiple drug resistance. Nanodelivery systems have emerged as the novel cancer therapeutics to overcome limitations of clinical use of anthraciclines. Doxil, a PEGylated liposome formulation of doxorubicin, was developed and approved by the FDA in 1995. The liposome is able to passively target anthracyclines into the tumor tissues via the EPR effect, escape from RES recognition, prolong circulation time in blood, and improve the drug distribution in the body. This formulation demonstrated slower plasma clearance, enhanced circulation and half-life, and decreased cardiotoxicity compared to free doxorubicin. Unfortunately, this formulation does not address the MDR problem. To date, many nanodelivery systems have been developed and reported and include liposomes, polymeric, solid lipid, mesoporous silica and magnetic nanoparticles, and polymer-drug conjugates to effectively circumvent MDR both in vitro and in vivo and some of these systems have even been advanced to clinical trials [33]. 
Vincristine and vinblastine are alkaloids from Catharanthus roseus (periwinkle), a plant of the Apocynaceae family. They are currently approved anticancer drugs inducing tubulin to form spiral polymers at physiological protein concentrations, thus interfering in the formation of microtubules. Vinblastine and vincristine are used for children's leukemia and in the treatment of Hodgkin's disease. In particular, vincristine sulphate is used to treat Wilm's tumor, neuroblastoma, breast cancer, rhabdomyosarcoma, and osteogenic sarcoma. In 2012, the FDA approved a nanoformulation of vincristine sulphate loaded in liposome (Marqibo) for the treatment of adult patients with Philadelphia chromosome-negative (Ph-) acute lymphoblastic leukemia. Vincristine sulphate administered as a liposomal formulation exhibits a lower clearance and higher AUC compared with conventional drug formulations. Other nanoformulations based on vincristine sulphate liposomes have been approved for the treatment of relapsed aggressive non-Hodgkin's lymphoma and other cancers. Advantages of liposomes over the existing conventional preparations are the increased blood circulation time, the intensification drug accumulation in the tumor, prolonged efficacy over an extended period [34,35].

Multifunctional nanoparticles, based on $\mathrm{pH}$-sensitive PLGAPEG-Fol and cell penetrating peptide $R_{7}$-conjugated PLGA-PEG, have been prepared for targeting vincristine sulphate to tumors and overcoming MDR. In this study, the $\mathrm{pH}$-triggered vincristine release was $65.6 \%$ during $8 \mathrm{~h}$ at $\mathrm{pH} 5.0$, but only $35.8 \%$ at $\mathrm{pH} 7.4$, demonstrating that a large amount of vincristine was released rapidly in a weak acidic environment. These innovative nanoparticles significantly enhance cellular uptake and cytotoxicity in MCF-7 and MCF-7/Adr cells when compared to the nanoparticles solely modified by Fol or $\mathrm{R}_{7}$. With Fol receptor-mediated endocytosis and strong intracellular penetration, vincristine-Fol/ $R_{7}$ NPs increased drug accumulation in resistant tumor cells by escaping P-glycoprotein-mediated drug efflux. In vivo imaging suggested the active targeting attributed to $\mathrm{pH}$ sensitivity, and the Fol receptor-mediated effect could improve tumor-targeting efficacy. Indeed, vincristine-Fol/R 7 NPs exhibited the strongest antitumor efficacy in vivo. Therefore, Fol/ $R_{7} N P s$ are effective nanocarriers for delivering antitumor drugs and overcoming MDR [36].

Camptothecin is a quinoline alkaloid derived from the bark, wood, and fruit of the Asian tree Camptotheca acuminata L. (Nyssaceae). Drs. Wall and Wani discovered camptothecin in 1966 and the National Cancer Institute investigated its potential antitumor activity as a selective inhibitor of DNA TOPO I. Renewed research led to the development of water-soluble analogues of camptothecin that also retained the anticancer activity, thus overcoming one of the major limitations of clinical use. Irinotecan and topotecan were approved in 1996 by the FDA for the treatment of colon, lung, breast, and ovarian cancers. Due to major limitations of these derivatives, several nanoparticulate formulations are currently being developed with the aim of improving the therapeutic index of these compounds. Lipid-based formulations of camptothecin, both liposomal and polymeric micelles, have been produced and tested for parenteral administration in the treatment of ovarian and colorectal cancers. These nanoformulations enable the delivery of higher amounts of drug to target tissues by virtue of the pharmacokinetic properties of the carrier and reduce toxicity to nontarget tissues [37,38].

\section{Artemisinin nanocarriers: improved water solubility, stability, and efficacy}

Artemisinin (qinghaosu) is a unique sesquiterpene lactone with an endoperoxide moiety isolated from Artemisia annua L. (qinghao, Asteraceae). Discovered by Youyou Tu in 1972 it is currently recommended by the WHO in combination with other antimalarial drugs to treat drug-resistant Plasmodium falciparum strains, cerebral malaria, and malaria in children. Beyond malaria, artemisinin and its derivatives (artemisinins) exert significant activities towards other protozoans (Leishmania, Trypanosoma, amoebas, Neospora caninum, and Eimeria tenella), trematodes (Schistosom a, liver flukes), and viruses (human cytomegalovirus, hepatitis B and C viruses). Recently, the activity of artemisinin and its derivatives against cancer, bacteria, and fungi have also been proved. The therapeutic value of artemisinins is limited due to poor water and oil solubility, a low bioavailability after oral administration because of rapid degradation by the liver, and a short (ca. $2.5 \mathrm{~h}$ ) halflife [39]. Therefore, there is an urgent need to develop new artemisinin formulations to increase its bioavailability, selectivity, and therapeutic application.

Artemisinin-loaded conventional and PEG (PEGylated) decorated liposomes were proposed as nanocarriers to increase biopharmaceutical properties of the drug. The pharmacokinetic profile and the main pharmacokinetic parameters of the carriers were evaluated in healthy mice after i.p. administration. Free artemisinin was rapidly cleared from plasma and hardly detected $1 \mathrm{~h}$ after administration. Conversely, both liposomal formulations showed a much longer blood circulation time than free artemisinin; artemisinin was still detectable after 3 and $24 \mathrm{~h}$ of administration, respectively, for conventional and PEGylated liposomes. $\mathrm{AUC}_{0-24} \mathrm{~h}$ values were increased by approximately six times in both liposomal formulations in comparison with free artemisinin. A strong effect of formulation on the half-life of artemisinin was enhanced by more than 5-fold by the incorporation of PEG into liposomes. Liposomes loaded with artemisinin, especially the long circulating vesicles, could really represent a new strategy for developing smart, well tolerated, and efficacious therapeutic nanocarriers to treat tumors, but it could also be very useful to treat parasitic diseases [39].

The antimalarial properties of artemisinin liposomes and artemisinin plus curcumin liposomes have also been investigated in Plasmodium berghei NK-65 infected mice, a suitable model for studying malaria because the infection has structural, physiological, and life cycle analogies with the human disease. Mice were treated with artemisinin at a dosage of $50 \mathrm{mg} / \mathrm{kg} /$ day or artemisinin plus curcumin administered at a dosage of $100 \mathrm{mg} / \mathrm{kg} /$ day. Artemisinin began to decrease parasitemia levels only 7 days after the start of the treatment and it appeared to have a fluctuant trend in blood concentration, which is reflected in the antimalarial effectiveness (Fig. 1S, Supporting Information). By contrast, treatments with $\mathrm{A}-\mathrm{CL}, \mathrm{AC}-\mathrm{CL}, \mathrm{A}-\mathrm{PL}$, and $\mathrm{AC}-\mathrm{PL}$ appeared to have an immediate antimalarial effect. All formulations cured malaria-infected mice within the same post-inoculation period. Additionally, all formulations showed less variability in artemisinin plasma con- 
centrations, which suggests that $\mathrm{A}-\mathrm{CL}, \mathrm{AC}-\mathrm{CL}, \mathrm{A}-\mathrm{PL}$, and $\mathrm{AC}-\mathrm{PL}$ give a modified release of the drug(s) and, consequently, a constant antimalarial effect during time. In particular, A-PL seems to give the most pronounced and statistically significant therapeutic effect in this murine model of malaria. The enhanced permanency in the blood of A-PL suggests the use of these nanosystems as suitable passive targeted carriers for parasitic infections [40].

In a further study by the same scientists, dihydroartemisinin, one of the most potent anticancer artemisinin-like compounds due to its apoptotic effects, was formulated in liposomes. Besides its effectiveness, dihydroartemisinin is a poorly water-soluble drug with low bioavailability and a low half-life (34-90 min), therefore, the development of new formulations of dihydroartemisinin that enable quick availability to the body is in great need. Conventional and PEGylated liposomes were loaded with dihydroartemisinin. A higher internalization occurred in the conventional formulation with respect to the stealth one, suggesting that the hydrophilic steric barrier of PEG molecules can reduce cellular uptake. Flow cytometry analysis was used also an alternative technique for rapid size determination of liposomes. Cytotoxicity studies in the MCF-7 cell line confirmed the absence of toxicity in blank formulations and suggested that liposomes may be suitable carriers for delivery of DHA, avoiding the use of formulations based on organic solvents [41].

The same group of researchers investigated the antitumor properties of artemisinin using a novel actively targeted nanocarrier, a PEGylated nanoliposome decorated with transferrin. Hence, transferrin receptors are largely expressed in cancer cells where the iron content is higher than in normal cells. Artemisinin-loaded liposomes were investigated for their cell uptake and cytotoxicity properties (Fig. 2S, Supporting Information) using the HCT-8 cell line, selected among several cell lines because of transferrin receptor overexpression. The results confirmed the enhanced delivery of artemisinin loaded in liposomes actively targeted with transferrin in comparison with the other artemisinin-loaded liposomes and an improved cytotoxicity [42].

A recent review of artemisinin-based nanocarriers describes liposomes, niosomes, micelles, solid lipid nanocarriers, nanostructured lipid carriers, nanoparticles, fullerenes, and nanotubes with different therapeutic applications. These nanovectors offer significant promise in improving the half-life and controlled release, better permeability, resistance to metabolic modification, and highly specific site targeted delivery [43].

\section{Curcumin-loaded nanoparticles: increased efficacy of a plethora of activities}

Curcumin is the major bioactive constituent of the rhizome of turmeric (Curcuma longa L., Zingiberaceae), an Indian traditional herbal medicine. Isolated in 1815 , its chemical structure, determined in 1910, is diferuloylmethane [1,7-bis(4-hydroxy-3-methoxyphenyl)-1,6-heptadiene-3, 5 dione] and it possesses antiangiogenic, antiproliferative, antitumorigenic, antioxidant, and anti-inflammatory properties in both in vitro and in vivo studies. Due to its multitargeting ability in various pathological conditions, it is very stimulating in its translation into a therapeutic agent. Like many other lipophilic small molecules, curcumin also has limitations for its efficient use in clinical scenarios in order to treat disease conditions. These include the low hydrophilicity and intrinsic dissolution rate(s), low physical and chemical instability, rapid metabolization with low nanomolar levels of the parent compound and its glucuronide and sulphate conjugates found in the peripheral or portal circulation, low absorption, poor pharmacokinetics and bioavailability, and low penetration and targeting efficacy.

During the last decade, an extraordinary and astonishing number (ca. one thousand) of nanoformulations have been undertaken to enhance curcumin use in in vitro, in vivo, preclinical, and clinical settings that involve the use of nanogels, and a plethora of lipid and hydrophilic nanocarriers, i.e., polymeric nanoparticles, liposomes, micelles, nanoemulsions, CD complexes, nanodisks, nanofibers, solid lipid nanoparticles, and solid nanodispersions. The specific roles and advantages of each delivery system are reported in a very comprehensive and recent review [44]. Many of these efforts initially dealt with improved bioavailability, but newer formulations pay attention to efficient targeting of curcumin at the diseased area with the aid of antibody, aptamer, and peptide mediation [44].

Biodegradable PEGylated polyester nanoparticles loaded with curcumin were investigated for their bioavailability using a Caco2 cell model to characterize the cellular transport pathway and determine the effect of polymer architecture including PEG chain length and core material on its cellular interaction and transcellular transport. PEG chain length (from 2000 to 5000 Da) and core material (PLA/PLGA) hardly affected the cellular interaction and the intracellular itinerary of the nanoparticles. However, in the case of transcellular transport, the maximal transcellular transport efficiency for its payload was achieved by the PEG5000PLA40000 nanoparticles, which present higher drug loading capacity and slower drug release. The findings revealed the cellular interaction mechanism of PEGylated polyester nanoparticles and provided evidence for the role of polymer architectures in modulating the transcellular permeability of the agents loaded by the nanoparticles, and would be helpful in improving carrier design to enhance drug delivery [45]. Kundu et al. [46] investigated antiglioma activity of curcumin-loaded lipid nanoparticles and its enhanced bioavailability in brain tissue for effective glioblastoma therapy. Curcumin-loaded nanoparticles inhibited cellular proliferation, migration, and invasion along with a higher percentage of cell cycle arrest and telomerase inhibition, leading to a greater percentage of apoptotic cell death in glioma cells compared with native curcumin. An in vivo study demonstrated an enhanced bioavailability of curcumin in blood serum and brain tissue when being delivered by curcumin-loaded glyceryl monooleate nanoparticles compared with native curcumin in a rat model. A study performed by Kakkar et al. [47] pursued to improve the oral bioavailability of curcumin by incorporating it into solid lipid nanoparticles. The developed nanoparticles (size ca. $130 \mathrm{~nm}$ ) exhibited prolonged drug release in vitro. In vivo pharmacokinetic studies revealed that after oral administration $(50 \mathrm{mg} / \mathrm{kg}, 25 \mathrm{mg} / \mathrm{kg}$, $12.5 \mathrm{mg} / \mathrm{kg}$, and $1 \mathrm{mg} / \mathrm{kg}$ ), a significant improvement in oral bioavailability was achieved by the nanoparticles compared to free curcumin (by 39, 32, 59, and 155 times at $50 \mathrm{mg} / \mathrm{kg}, 25 \mathrm{mg} / \mathrm{kg}$, $12.5 \mathrm{mg} / \mathrm{kg}$, and $1 \mathrm{mg} / \mathrm{kg}$ doses, respectively). An in vitro test, the so-called parallel artificial membrane permeability assay 
(PAMPA), has also been reported to evaluate permeation of curcumin loaded in different nanocarriers developed for oral administration. Righeschi et al. [48] focused the studies on the development and characterization of solid lipid nanoparticles for the encapsulation of curcumin for oral administration using GRAS excipients, i.e., Compritol and lecithin. The particle size was lower than $300 \mathrm{~nm}$ with a drug entrapment efficiency of $80 \%$. PAMPA tests showed a considerable increase of curcumin permeated when formulated as solid lipid nanoparticles. X-ray studies and a modified release profile suggested that curcumin molecules are solubilized into the solid lipid matrix. A further approach to improve the solubility and the stability and oral uptake of curcumin was the formulation of o/w microemulsions using food grade components. Three microemulsions were developed and characterized, stabilized by non-ionic surfactants Cremophor EL, Tween 20 , Tween 80 , or lecithin and contained a variety of oils, namely, olive oil, wheat germ oil, and vitamin E. The oral absorption of curcumin microemulsions was investigated in vitro using PAMPA. The optimal formulation consisted of vitamin E (3.3 g/100 g), Tween $20(53.8 \mathrm{~g} / 100 \mathrm{~g})$, ethanol $(6.6 \mathrm{~g} / 100 \mathrm{~g})$, and water $(36.3 \mathrm{~g} /$ $100 \mathrm{~g}$ ), obtaining a percentage of permeation through the artificial membrane of about $70 \%$ [49]. Dendrosomal curcumin, a polymeric micelle of $142 \mathrm{~nm}$ with constant physical and chemical stability, synthesized by esterification of oleoyl chloride $(0.01 \mathrm{~mol})$ and polyethylene glycol 400 (0.01 mol) was developed and tested for the inhibition on the proliferation of U87MG cells, a cellular model of glioblastoma. Evaluation was made by considering master genes of pluripotency and regulatory miRNA. Methylthiazol tetrazolium assay and flow cytometry were used to detect the antiproliferative effects of dendrosomal curcumin. Annexin-VFLUOS and caspase assay were used to quantify apoptosis. The sensitivity of U87MG cells as well as hBMSC and HFSF-PI3 cells to DNC, free curcumin, and empty dendrosome were studied using MTT assay. The nanoparticle significantly suppressed the proliferation of U87MG cells in a time- and dose-dependent manner (Fig.3S, Supporting Information). The half maximal inhibitory concentration $\left(\mathrm{IC}_{50}\right)$ of dendrosoma for U87MG cells was $20 \mu \mathrm{M}$ after $24 \mathrm{~h}$ (Fig. $35 \mathrm{~A}$, Supporting Information) and $48 \mathrm{~h}$ (Fig. 3S B, Supporting Information), which declined to $10 \mu \mathrm{M}$ at $72 \mathrm{~h}$ ( $p<0.001$ ) (Fig. 3S C, Supporting Information). However, the viability of U87MG was not affected by free curcumin only at $72 \mathrm{~h}$. Dendrosome increased the water solubility and entry of curcumin to cells without any toxic effects. As indicated in Fig. S3, Supporting Information, dendrosomes induced apoptosis in a time- and dose-dependent manner. Studying the inhibitory effects of dendrosome on hBMSC showed that these cells are affected by the nanocarrier as well as cancerous cells and in a concentration higher than in U87MG cells. Fig. 4S A, Supporting Information, shows the effects of dendrosome on cell viability of hBMSC after $24 \mathrm{~h}$ of drug exposure. Cell viability declined to $67 \%$ after treatment with $25 \mu \mathrm{M}$ dendrosome $(\mathrm{p}<0.01)$ and to 35 and $31 \%$ after treatment with $30 \mu \mathrm{M}$ and $35 \mu \mathrm{M}$ dendrosome, respectively $(\mathrm{p}<0.001)$. The loss of cell viability was also determined after 48 hours of treatment with DNC (Fig. 4S B, Supporting Information). The viability of HFSF-PI3 was not significantly affected due to treatment and at determined $\mathrm{IC}_{50}$ for U87MG lines, no inhibitory effect was detected on these cells ( $p>0.05)$; however, at $25 \mu \mathrm{M}$ concentration, the viability of the cells decreased to $50 \%$ (Fig. 5S, Supporting Information) $(p<0.01)$. Therefore, in concentrations suppressive for cancer cells, no harmful effects connected to dendrosome were observed in stem cells and normal fibroblast cells, showing the safety of this formulation as an anticancer treatment agent on normal cells [50]. Finally, Bhawana et al. [51] developed curcumin-loaded polymeric nanoparticles, $(2-40 \mathrm{~nm})$ with antimicrobial activity. Efficacy was evaluated using a microplate dilution technique against Streptococcus aureus, Bacillus subtilis, Escherichia coli, Pseudomonas aeruginosa, Penicillium notatum, and Aspergillum niger. The water solubility and small size of curcumin nanoparticles enhanced antimicrobial activity when compared to free curcumin. The antibacterial activity was more pronounced than its antifungal activity. Among bacteria, gram-positive strains were more sensitive. TEM analysis revealed that when the nanoparticles were introduced into a bacterium, they completely destroyed the cell wall, resulting in bacterial cell death. A recent review reported the therapeutic application of different natural products, mainly polyphenols, including curcumin in rheumatoid arthritis therapy. The review highlighted how nano/submicron vectors can be useful in increasing bioavailability and efficacy of the natural products [52].

\section{Resveratrol-loaded nanocarriers to improve stability and efficacy}

Resveratrol (3,4',5-trihydroxy-trans-stilbene) is a natural polyphenolic compound abundant in grapes, peanuts, and red wine. First detected in grape vines (Vitis vinifera L., Vitaceae), especially when infected with Botrytis cinerea, resveratrol is synthesized almost entirely in the skin and its content is at its maximum just before the grapes reach maturity. Therefore, resveratrol's highest concentration is in the skin and seeds of grapes (50-100 $\mu \mathrm{g}$ per gram, corresponding to $5-10 \%$ of their biomass). Like the majority of polyphenols, it has a rapid and extensive metabolism with the formation of several resveratrol glucuronides and sulphates. In humans, about $70 \%$ of orally administered resveratrol $(25 \mathrm{mg})$ is rapidly (<30 min) absorbed and metabolized, with a peak plasma level of ca. $2 \mu \mathrm{M}$ of resveratrol metabolites and a half-life of 9$10 \mathrm{~h}$. Furthermore, there is a significant person-to-person variability in drug absorption and metabolic processes. The extent to which the human colon can absorb and metabolize resveratrol depends on the hepatic function and on the metabolic activity of the local intestinal microflora [53].

Resveratrol has emerged as one of the most promising naturally occurring compounds with great preventive and therapeutic efficacies. Studies have confirmed its potential in the prevention of coronary disease and neurodegenerative pathologies. Resveratrol has been reported to elicit many cellular responses, including cell cycle arrest, differentiation, and apoptosis, and to inhibit the growth of several types of cancer, principally prostate and colon cancers. Resveratrol and its analogues present pharmacological safety and may be used in combination with other agents to enhance therapeutic efficacy and minimize toxicity. However, intrinsic features that lead to low bioavailability, low water solubility, and instability have compromised its wide array of activity, and numerous nanoformulations have been currently investigated to enhance its efficacy. 
Shao et al. [54] developed mPEG poly(epsiloncaprolactone)based nanoparticles incorporating resveratrol and demonstrated a significantly higher rate of cell death as compared to an equivalent dose of free resveratrol in glioma cells. Resveratrol loaded in solid lipid nanoparticles resulted in decreasing cell proliferation, preventing skin cancer [54]. In another study, resveratrol nanoparticles based on poly(epsiloncaprolactone) and poly(D,L-lacticco-glycolic acid)-poly(ethylene glycol) were proven to strongly enhance the uptake by PCa cell lines. In addition, when the PCa, DU145 , and LNCaP cell lines were treated with the nanosystems, all three cell lines showed significantly elevated cytotoxicity compared to those treated with free resveratrol at different concentrations (from $10 \mu \mathrm{M}$ to $40 \mu \mathrm{M}$ ). Remarkably, there was a consistent sensitivity of nano-resveratrol towards both the hormone sensitive LNCaP cells and androgen-independent DU-145 prostate cancer cell lines [55].

In a further study, mitochondrial targeting resveratrol liposomes modified with a dequalinium polyethylene glycol-distearoylphosphatidyl ethanolamine conjugate were developed. The nanopartices induced apoptosis in both nonresistant and resistant cancer cells by the dissipating mitochondrial membrane potential. It also increased caspase- 9 and caspase- 3 activities. Significant antitumor efficacy was exerted by resveratrol liposomes in xenografted resistant A549/cDDP cancers in nude mice and tumor spheroids by deep penetration [56]. In another study, the viability of HEK 293 cells and their photoprotection after UV-B irradiation was tested with free and liposomal resveratrol. Interestingly, cell viability was found to be decreased at a $100 \mu \mathrm{M}$ concentration, and cell proliferation increased at $10 \mu \mathrm{M}$ and achieved the most effective photoprotection. This study showed the effectiveness of resveratrol at $10 \mu \mathrm{M}$ and also toxicity at higher concentrations considering the changes in apoptotic features and cell shape and its detachment [57].

The trans-resveratrol-loaded lipid core nanocapsules were used to test its antiglioma activity on the C6 glioma cell line in vitro and on brain implanted C6 cells in in vivo models. In vitro studies indicated that lipid nanostructured carriers loaded with resveratrol decreased the cell viability of C6 glioma cells to a much greater extent when compared to resveratrol used in solution. In vivo studies of the nanoparticles also showed a marked decrease in the size of the tumor, suggesting they could be used effectively in the treatment of gliomas [58]. A further study was carried out on several anti-inflammatory natural products, namely, carvacrol, thymol, resveratrol, pterostilbene, $\mathrm{N}$-(3-oxo-dodecanoyl)-I-homoserine lactone, caffeic acid, and caffeic acid phenethyl ester. All of the constituents were loaded in liposomes to improve their solubility, stability, and bioavailability, therefore addressing the particular challenges associated with each of these natural agents. The chemical instability of resveratrol was improved by liposome encapsulation, preventing the inactivation of cis-trans isomerization, and its intravenous administration inhibited tumor growth by approximately $70 \%$ in a murine tumor model, showing that simple solubilization can have important therapeutic benefits [59].

\section{Nanovectors loaded with salvianolic acid: exploring other activities}

Salvianolic acid B represents the most characteristic hydrophilic constituent, Salvia miltiorrhiza Bunge (Lamiaceae), a very famous herbal drug of traditional Chinese medicine. In virtue of the few side effects, extracts and single constituents are successfully used in clinics in China, Korea, Japan, and other Asian countries for the treatment of heart and cerebrovascular disease, hepatitis, hepatocirrhosis, neurasthenic insomnia, cancer, chronic renal failure, and dysmenorrhea. Both extracts and single constituents have positive effects in CNS neuronal injury and degeneration in several animal models [60].

Strong antioxidant activity of salvianolic acid B has also been reported and it may be useful in the treatment of some severe chronic diseases where there is an imbalance of reactive oxygen species formation and where intracellular reactive oxygen and nitrogen species levels can cause severe cell damage and even cell death. In particular, it can protect against oxidative stress as well as the antioxidant superoxide dismutase and reduced activity of glutathione, important determinants of neuropathological and behavioral consequences in neuropathic pain. This is a chronic disease defined by the WHO as an untreatable illness because therapeutics are unsatisfactory in many cases and there is an urgent need to discover and develop novel active drugs. Both conventional and long circulating liposomes loaded with salvianolic acid B were developed for parental administration due to the poor chemical stability and bioavailability of salvianolic acid B. These carriers were submitted to pharmacological studies using the paw pressure test in an animal model of neuropathic pain where a peripheral mononeuropathy was produced by a chronic constriction injury of the sciatic nerve. Salvianolic acid B was effective against mechanical hyperalgesia when administered intraperitoneally at a dose of $100 \mathrm{mg} / \mathrm{kg}, 15 \mathrm{~min}$ after administration. According to the in vivo studies, encapsulation, especially into PEGylated liposomes, increased and prolonged the antihyperalgesic activity 30 min after i. p. administration and the effect was still significant after $45 \mathrm{~min}$ [61].

\section{Honokiol loaded in nanocarriers: retarded elimination and prolonged residence time in the circulating system}

Honokiol, 3',5-di(2-propenyl)-1,1'-biphenyl-2,4'-diol, is a constituent of the Chinese medicinal plant Magnolia officinalis L. (Magnoliaceae). It has several pharmacological effects, including anti-inflammatory, antithrombotic, antirheumatic, and antioxidant with anxiolytic, central nervous system depressant, and muscle relaxant activities. In addition, it has potent antitumor activity. Again, this compound's hydrophobic properties represent an obstacle, because high hydrophobicity prevents vascular administration. Stable liposomes containing an inclusion complex of honokiol in $\mathrm{HP}-\beta-\mathrm{CD}$ used a molar ratio of honokiol/HP- $\beta-\mathrm{CD} /$ phosphatidil choline $1: 2: 2$. The mean particle size was $123.5 \mathrm{~nm}$, the zeta potential was $-25.6 \mathrm{mV}$, and the EE was $91.09 \pm 2.76 \%$. The release profile in vitro demonstrated that honokiol is released from the liposome with a sustained and slow profile. A pharmacokinetic study revealed that honokiol-in-HP- $\beta$-CD-in-liposome significantly retarded the elimination of honokiol and prolonged the residence 
time in the circulating system. Honokiol-in-HP- $\beta$-CD-in-liposome had antiproliferative activity in A549 and HepG2 tumor cells compared with free honokiol [62].

In a further study, self-assembled pectin nanoparticles containing a complex of HP- $\beta$-CD and honokiol were prepared and evaluated for delivering honokiol to HepG2 cells. An investigation of the in vitro release indicated that the drug-loaded nanoparticles exhibited a higher drug release rate than free honokiol and an effective sustained release. Cytotoxicity, cell apoptosis, and cellular uptake studies further confirmed that the pectin nanoparticles with galactose residues generated higher cytotoxicity than free honokiol on HepG2 cells, which highly expressed ASGR. Nevertheless, these findings were not observed in ASGR-negative A549 cells under similar conditions. Therefore, pectin nanoparticles loaded with honokiol complexed with HP- $\beta$-CD have a specific active targeting ability to ASGR-positive HepG2 cells and could be used as potential drug carriers in the treatment of liver-related tumors [63].

Nanoparticles loaded with honokiol were prepared with thermosensitive poly(ethylene glycol)-poly(epsilon-caprolactone)poly(ethylene glycol) hydrogel to improve honokiol therapeutic efficacy on malignant pleural effusion-bearing mice. The hydrogel was administered intrapleurally after Lewis lung carcinoma cell inoculation. Honokiol-hydrogel reduced the number of pleural tumor foci, prolonging the survival time of MPE-bearing mice more effectively compared with the control groups. In addition, honokiol-hydrogel successfully inhibited angiogenesis as assessed by CD31 ( $p$ 0.05). Histological analysis of pleural tumors exhibited that honokiol loaded in hydrogel led to an increased rate of apoptosis [64].

PEGylated liposomal honokiol with a particle size of ca. $100 \mathrm{~nm}$ has been developed, and both pharmacokinetic properties and human plasma protein binding ability were investigated. The pharmacokinetics properties were studied after intravenous administration in Balb/c mice. There were significant differences of $\mathrm{t}(1 / 2 \beta)$ and $\mathrm{AUC}(0 \rightarrow \infty)$ parameters. The mean $\mathrm{t}(1 / 2 \beta)$ value of PEGylated liposomal honokiol and free honokiol were $26.09 \mathrm{~min}$ and $13.46 \mathrm{~min}$, respectively. The $\mathrm{AUC}(0 \rightarrow \infty)$ ratio of PEGylated liposomal honokiol to free honokiol was about 1.85-fold (219.24/ $118.68 \mu \mathrm{g} / \mathrm{mL} \mathrm{min})$. Honokiol at concentrations of $0.5,8.0$, and $20 \mu \mathrm{g} / \mathrm{mL}$ in human plasma achieved a bound percentage from 60 to $65 \%$. The results suggested that PEGylated liposome improved honokiol solubility, increased the drug concentration in plasma, and decreased the clearance [65].

\section{Flavonoids loaded in nanocarriers to enhance bioefficacy}

Flavonoids are among the biggest group of polyphenols, widely distributed in plants, especially in edible plants. Hence, flavonoids represent the active constituents of many dietary supplements and herbal remedies, as well as there is an increasing interest in this class of polyphenols as functional ingredients of beverages, food grains, and dairy products. A plethora of evidence supports the health benefits and value of flavonoids, which can play a role in physiological function treatment and in the prevention of diseases, particularly of degenerative conditions including cancers, cardiovascular, and neurodegenerative diseases. Conversely, vari- ous studies have also shown that flavonoids have some drawbacks after oral administration, principally stability, bioavailability, and bioefficacy.

Water solubility and gastric stability are the major limiting factors for flavonoids to pass the biological membrane and to be absorbed systematically following oral administration. An enormous number of nanodelivery techniques, including nanoparticles, nanocapsules, micro- and nanoemulsions, micelles, vesicles, solid lipid nanoparticles, and nanostructured lipid capsules, were successfully developed for overcoming the delivery challenges of flavonoids, as recently reported in a review [66]. Most of the papers are related to pure epigallocatechin gallate, quercetin, rutin, apigenin, baicalein, puerarin, apigenin, and hesperetin using principally biodegradable nanoparticles (natural or synthetic polymers), micelles, vesicles, and micro- and nanoemulsions. Results have shown that mucoadhesive chitosan nanoparticles loaded with flavonoids were very useful in increasing flavonoid bioavailability. In comparison to microdelivery systems, nanocarriers have some advantages: they are more stable, provide more surface area, and have the potential to enhance bioavailability by increasing the absorption from enterocytes, by receptor-mediated endocytosis and transcytosis, and by phagocytosis via specialized microfold cells. Finally, nanodelivery systems improve the controlled release of the encapsulated flavonoids. A recent review focused on nanocarriers made of GRAS approved compounds and represent driving formulating carriers for innovative and more effective formulations of functional foods, dietary supplements, and herbal medicinal products [16].

\section{Successful nanoformulations based on extracts}

Extracts are complex mixtures of constituents having different polarities and absorption properties and, in many cases, have good activity in in vitro assays, which is not reproducible in in vivo experiments. In some cases, constituents can be incompatible with other components in the formulation or have undesirable properties, which, consequently, nanotechnological strategies have attempted to enhance their efficacy. The nanosystems can increase selectivity and activity, protect against thermal- or photodegradation, reduce side effects, promote sustained release of active constituents, or reduce the required dose, generally resulting in improved activity. Some recent reviews have focused on the nanotechnology approach of extracts using different drug delivery systems based on nanotechnology, principally polymeric nanoparticles, solid lipid nanoparticles, liquid crystal systems, vesicles, nanoemulsions, and microemulsions, in order to impart a formulation's most desirable properties. The most investigated are the extracts of ginseng, green tea, ginkgo, and milk thistle [66-68].

\section{Nanocarriers based on sylimarin to optimize hepatic protection}

Silymarin is one of the best-known hepatoprotective extracts obtained from the seeds of Silybum marianum L., commonly known as milk thistle (Asteraceae). Since ancient times, this plant has been used as an herbal cure for liver and biliary tract diseases. It has been reputed that milk thistle extracts safeguard and regenerate liver cells in various diseases affecting liver, principally cirrhosis, jaundice, and hepatitis. It exhibits strong antioxidant ac- 
tion via free radical scavenging activity and inhibits lipid peroxidation. It also prevents the entry of harmful toxicants, primarily heavy metals, pesticides, alcohols, medicines, and $\mathrm{CCl}_{4}$ in the liver, thereby protecting the liver cells from further damage. Silymarin is a mixture of flavonolignans, which comprises silybin, isosilybin, silydianin, and silychristine. It is poorly soluble in water and exerts its hepatoprotective action at an oral dose of $240-800 \mathrm{mg} /$ day in two or three divided doses. When administered orally, peak plasma concentration is reached in $2-4 \mathrm{~h}$, with $\mathrm{t}_{1 / 2}=6 \mathrm{~h}$. Only $20-$ $50 \%$ silymarin is absorbed from the gastrointestinal tract where it undergoes extensive enterohepatic circulation. Therefore, absorption of silymarin from the gastrointestinal tract is low, making bioavailability poor. Its poor bioavailability is mainly due to extensive metabolism, poor aqueous solubility, and rapid excretion through urine and bile as well as low permeability across intestinal epithelial cells. Silymarin represents a good candidate for nanoformulations. In 2011, a very interesting review concerning the recent published literature on various techniques for increasing the bioavailability of silymarin, including the nanotechnological approach, was published [69]. A more recent study reported that silymarin-loaded solid nanoparticles, containing silymarin/castor oil/polyvinylpyrrolidone/Transcutol HP/Tween 80 at a weight ratio of 5/3/3/1.25/1.25, improved drug solubility about 1300 -fold [70]. Additionally, after oral administration of $10 \mathrm{mg} / \mathrm{kg}$ in rats, the nanoparticles showed a considerably shorter time to peak concentration. In particular, the plasma concentrations of silymarin in nanoparticles between 0.25 and $1.5 \mathrm{~h}$ were impressively higher when compared with a silymarin conventional formulation (1.27 and 1.44 times, respectively, $p<0.05$ ). The AUC of the drug provided by the nanoparticles was approximately 1.3 -fold greater than that of the conventional formulation. On the other hand, $T_{\max }$ for the nanoparticles was significantly shorter than that of the conventional formulation $(p<0.05)$. The plasma levels achieved by the nanoparticles increased up to $22.85 \pm 3.59 \mu \mathrm{g} /$ $\mathrm{mL}$ at $\mathrm{T}_{\max }(1 \mathrm{~h})$. Therefore, the enhanced oral bioavailability of silymarin in rats might be associated with a marked enhancement in the absorption rate of silymarin as a result of the increased dissolution of the drug in the nanoparticles. In addition, the silymarin-loaded nanoparticles significantly reduced $\mathrm{CCl}_{4}$-induced hepatotoxicity, indicating improved bioactivity compared with silymarin conventional formulation and the pure drug. Treatment with $\mathrm{CCl}_{4}$ significantly elevated the SAT activity, which was significantly reduced by pretreatment with silymarin-loaded nanoparticles. The protective effects of silymarin-loaded nanoparticles on $\mathrm{CCl}_{4}$ induced acute liver toxicity were also observed by histopathologic examination. Compared with healthy livers, which had normal lobular architecture with a central vein and radiating hepatic cords, liver sections from $\mathrm{CCl}_{4}$-exposed rats showed necrosis of hepatocytes, hepatocyte degeneration, and infiltration of inflammatory cells. While the $\mathrm{CCl}_{4}$-induced destruction of liver architecture was not improved in rats treated with silymarin powder or the conventional formulation, the liver tissue from rats treated with silymarin nanoparticles showed less severe damage surrounding the central vein, suggesting superior hepatoprotective activity of silymarin nanoparticles over the drug powder or the commercial conventional formulation [70].

\section{Nanocarriers based on Cuscuta chinensis extracts}

Cuscuta chinensis Lam. (Convolvulaceae) is a commonly used traditional Chinese medicine to nourish the liver and kidney. The ethanolic extract is generally administered, but due to the poor water solubility of its major and characteristic constituents, flavonoids and lignans, its absorption upon oral administration could be limited.

Nanoparticles based on the ethanolic extract were developed and evaluated for the hepatoprotective and antioxidant effects on acetaminophen-induced hepatotoxicity in rats. An oral dose of the extract at 125 and $250 \mathrm{mg} / \mathrm{kg}$ and the extract loaded in the nanoparticles at 25 and $50 \mathrm{mg} / \mathrm{kg}$ showed a significant hepatoprotective effect relatively to the same extent $(p<0.05)$ by reducing levels of AST, ALT, and AP. These biochemical assessments were supported by rat hepatic biopsy examinations. In addition, the antioxidant activities of the extract and the nanoparticles both significantly increased superoxide dismutase, catalase, and glutathione peroxidase, and reduced malondialdehyde $(p<0.05)$. Moreover, the results also indicated that the hepatoprotective and antioxidant effects of $50 \mathrm{mg} / \mathrm{kg}$ of the extract loaded in the nanoparticles were effectively better than $125 \mathrm{mg} / \mathrm{kg}$ of the extract $(p<0.05)$. An oral dose of the extract loaded in nanoparticles, five times less as the extract itself, could exhibit similar levels of outcomes [71].

\section{Nanocarriers loaded with essential oils to optimize their therapeutic use}

Essential oils are complex blends of a variety of volatile molecules including terpenoids, phenol components, and aliphatic constituents, which have a strong interest in the pharmaceutical, sanitary, cosmetic, agricultural, and food industries. Since the Middle Ages, essential oils have been widely used for their bactericidal, virucidal, fungicidal, antiparasitical, insecticidal, and medicinal properties including analgesic, sedative, anti-inflammatory, spasmolytic, and locally anesthetic remedies. Their nanoencapsulation in drug delivery systems has been proposed to have the capability of decreasing volatility and improving stability, water solubility, and efficacy of essential oil-based formulations by maintenance of therapeutic effectiveness. Two categories of nanocarriers can be proposed: polymeric nanoparticulate formulations, extensively studied with significant improvement of the essential oil antimicrobial activity, and lipid carriers, including liposomes, solid lipid nanoparticles, nanostructured lipid particles, and nano- and microemulsions.

A significantly large part of the current literature on the encapsulation of essential oils deals with micrometric size capsules, which are used for the protection of the active compounds against environmental factors (e.g., oxygen, light, moisture, and $\mathrm{pH}$ ), to decrease oil volatility, and to transform the oil into a powder. Encapsulation in nanometric particles is an alternative for overcoming these problems but, additionally, due to the subcellular size, may increase the cellular absorption mechanisms and increase bioefficacy, as reported in a comprehensive recent review [17].

Among the different studies described in the literature, the effect of liposomal inclusion on the stability and in vitro antiherpetic activity of Santolina insularis L. (Asteraceae) essential oil was very 
remarkable. Pure essential oil cannot be use topically because of its high volatility and enhancer penetration properties, which can result in safety concerns, hence, a suitable formulation should be prepared. Liposomes successfully prevented its degradation and were effective in inactivating HSV-1, and that activity is principally due to direct virucidal effects [72].

The same authors also studied the antiherpetic activity of the essential oil of Artemisia arborescens L. (Asteraceae) loaded in liposomes. These carriers were very suitable to be incorporated in good amounts of essential oil, and stability studies pointed out that vesicle dispersions were very stable for at least six months. Neither oil leakage nor vesicle size alteration occurred during this period. After one year of storage, oil retention was still good, but vesicle fusion was present. Antiviral assays demonstrated that the liposomal formulation enhanced its in vitro antiherpetic activity [73].

In a further study, the same author prepared solid lipid nanoparticles loaded with $A$. arborescens essential oil and tested the antiherpetic activity. The nanoparticles entrapped the essential oil in high yields and the mean particle size increased only slightly after two years of storage, indicating a high physical stability. In vitro antiviral assays showed that nanoparticle incorporation did not affect the essential oil antiherpetic activity. The in vitro skin permeation experiments demonstrated the capability of nanoparticles of greatly improving the oil accumulation into the skin. Oil permeation occurred only when the oil was delivered from the control solution [74].

Frankincense and myrrh are gum resins obtained from the genera Boswellia and Commiphora, respectively. Modern pharmacological research has revealed that essential oils are the primary effective components in frankincense and myrrh oil that exhibit a broad spectrum of biological activities, principally antimicrobial, anti-inflammatory, and antitumor activities. As with other essential oils, the instability and poor water solubility result in poor oral bioavailability, which limits its clinical application. The components of frankincense and myrrh oil are very sensitive to light, air, and high temperature, and they stimulate the gastrointestinal tract, making these oils unsuitable for oral administration. A recent study has reported the successful preparation of solid lipid nanoparticles for the oral delivery of frankincense and myrrh essential oils [75].

\section{Concluding Remarks}

Extensive research is going on in natural products and herbal extracts to incorporate them into nano-drug delivery systems. The application of nanotechnology to drug delivery has already had a significant impact on many areas of medicine, and this approach is essential to developing appropriate therapeutic treatments of some essential drugs, principally antitumor and antiparasistic agents. Amazing nanoparticles loaded with paclitaxel, doxorubicin, vincristine, camptothecin, artemisinin, curcumin, resveratrol, honokiol, salvianolic acid B, green tea catechins, and silymarin are available on the market or are under clinical studies. Remarkable results have also been obtained for nanoformulations based on complex mixtures of constituents, extracts, and essential oils, which may extend a formulation's action, and successfully combine active substances with different degrees of hydrophilicity/ lipophilicity. So far, the results obtained from nanoencapsulated natural products are very encouraging, generally having better stability, sustained release, and improved bioavailability at much lower doses, and increasing long-term safety of these constituents. We have entered the era of nanomedicine with natural drugs, which is probably the most salient feature of the field and a decisive step toward the demonstration that natural drugs can continue to play a key role in modern clinics.

\section{Supporting information}

Data which represent parasitemia progression for mice groups infected with $P$. berghei and treated with ART loaded in lipososmes, in vitro cytotoxicity of free ART, ART-L, and ART-L-Tf on HCT-8 COIon cancer cells, and the effects of dendrosomal curcumin, free curcumin, and empty dendrosome on the viability of U87MG cells, human bone marrow-derived stem cells, and HFSF-PI3 cells are available as Supporting Information.

\section{Acknowledgements}

The authors wish to thank Ente Cassa Risparmio di Firenze for kindly supporting part of the research.

\section{Conflict of Interest}

The authors declare no conflict of interest.

\section{References}

[1] Cameron A, Ewen M, Auton M, Abegunde D. WHO (World Health Organization): The world medicines situation 2011: Medicines prices, availability and affordability. Geneva, Switzerland. Available at http://www. who.int/medicines/areas/policy/world_medicines_situation/WMS_ch6_ wPricing_v6.pdf. Accessed September 3, 2016

[2] Beghyn T, Deprez-Poulain R, Willand N, Folleas B, Deprez B. Natural compounds: leads or ideas? Bioinspired molecules for drug discovery. Chem Biol Drug Des 2008; 72: 3-15

[3] Lu JJ, Pan W, Hu YJ, Wang YT. Multi-target drugs: the trend of drug research and development. PLoS One 2012; 7: e40262

[4] Morphy R. Selectively nonselective kinase inhibition: striking the right balance. J Med Chem 2009; 53: 1413-1437

[5] Mehta P, Shah R, Lohidasan S, Mahadik KR. Pharmacokinetic profile of phytoconstituent $(\mathrm{s})$ isolated from medicinal plants - A comprehensive review. J Tradit Complement Med 2015; 5: 207-227

[6] Kesarwani K, Gupta R. Bioavailability enhancers of herbal origin: an overview. Asian Pac J Trop Biomed 2013; 3: 253-266

[7] Couvreur P, Vauthier C. Nanotechnology: intelligent design to treat complex disease. Pharm Res 2006; 23: 1417-1450

[8] Committee for proprietary medicinal products (CPMP). Note for guidance on the investigation of bioavailability and bioequivalence. Available at http://www.ema.europa.eu/docs/en_GB/document_ library/Scientific_guideline/2010/01/WC500070039.pdf. Accessed September 3, 2016

[9] Wu CY, Benet LZ. Predicting drug disposition via application of BCS: transport/absorption/elimination interplay and development of a bio- 
pharmaceutics drug disposition classification system. Pharm Res 2005; 22: $11-23$

[10] Fleisher D, Bong R, Stewart BH. Improved oral drug delivery: solubility limitations overcome by the use of prodrugs. Adv Drug Deliv Rev 1996; 19: $115-130$

[11] Stella V], Nti-Addae KW. Prodrug strategies to overcome poor water solubility. Adv Drug Deliv Rev 2007; 59: 677-694

[12] Merisko-Liversidge E, Liversidge GG, Cooper ER. Nanosizing: a formulation approach for poorly-water-soluble compounds. Eur J Pharm Sci 2003; 18: 113-120

[13] Möschwitzer JP. Drug nanocrystals in the commercial pharmaceutical development process. Int J Pharm 2013; 453: 142-156

[14] Hans ML, Lowman AM. Biodegradable nanoparticles for drug delivery and targeting. Curr Opin Solid State Mater Sci 2002; 6: 319-327

[15] Jabr-Milane LS, van Vlerken LE, Yadav S, Amiji MM. Multi-functional nanocarriers to overcome tumor drug resistance. Cancer Treat Rev 2008; 34: 592-602

[16] Bilia AR, Isacchi B, Righeschi C, Guccione C, Bergonzi MC. Flavonoids loaded in nanocarriers: an opportunity to increase oral bioavailability and bioefficacy. Food Nutr Sci 2014; 5: 1212-1227

[17] Bilia AR, Guccione C, Isacchi B, Righeschi C, Firenzuoli F, Bergonzi MC. Essential oils loaded in nanosystems: a developing strategy for a successful therapeutic approach. Evid Based Complement Alternat Med 2014; 2014: 651593

[18] Gaumet M, Vargas A, Gurny R, Delie F. Nanoparticles for drug delivery: the need for precision in reporting particle size parameters. Eur J Pharm Biopharm 2008; 69: 1-9

[19] Nicolas ], Mura S, Brambilla D, Mackiewicz N, Couvreur P. Design, functionalization strategies and biomedical applications of targeted biodegradable/biocompatible polymer-based nanocarriers for drug delivery. Chem Soc Rev 2013; 42: 1147-1235

[20] Gelperina S, Kisich K, Iseman MD, Heifets L. The potential advantages of nanoparticle drug delivery systems in chemotherapy of tuberculosis. Am J Respir Crit Care Med 2005; 172: 1487-1490

[21] Kumar M, Misra A, Babbar AK, Mishra AK, Mishra P, Pathak K. Intranasal nanoemulsion based brain targeting drug delivery system of risperidone. Int J Pharm 2008; 358: 285-291

[22] Emerich DF, Thanos CG. The pinpoint promise of nanoparticle-based drug delivery and molecular diagnosis. Biomol Eng 2006; 23: 171-184

[23] Bawa R. Nanoparticle-based therapeutics in humans: a survey. Nanotechol Law Business 2008; 5: 135-155

[24] Zhang L, Gu FX, Chan JM, Wang AZ, Langer RS, Farokhzad OC. Nanoparticles in medicine: therapeutic applications and developments. Clin Pharmacol Ther 2008; 83: 761-769

[25] Mezei M, Gulasekharam V. Liposomes - a selective drug delivery system for the topical route of administration. Lotion dosage form. Life Sci 1980; 26: 1473-1477

[26] Cevc G. Transfersomes, liposomes and other lipid suspensions on the skin: permeation enhancement, vesicle penetration, and transdermal drug delivery. Crit Rev Ther Drug Carrier Syst 1996; 13: 257-388

[27] Gupta M, Agrawal U, Vyas SP. Nanocarrier-based topical drug delivery for the treatment of skin diseases. Expert Opin Drug Deliv 2012; 9: 783-804

[28] Ping M, Mumper RJ. Paclitaxel nano-delivery systems: a comprehensive review. J Nanomed Nanotechnol 2013; 18: 1000164

[29] Green MR, Manikhas GM, Orlov S, Afanasyev B, Makhson AM, Bhar P, Hawkins MJ. Abraxane, a novel cremophor-free, albumin-bound particle form of paclitaxel for the treatment of advanced non-small-cell lung cancer. Ann Oncol 2006; 17: 1263-1268

[30] Gradishar W]. Albumin-bound paclitaxel: a next-generation taxane. Expert Opin Pharmacother 2006; 7: 1041-1053

[31] Untch M, Jackisch C, Schneeweiss A, Conrad B, Aktas B, Denkert C, Eidtmann $\mathrm{H}$, Wiebringhaus $\mathrm{H}$, Kümmel S, Hilfrich J, Warm M, Paepke S,
Just M, Hanusch C, Hackmann J, Blohmer JU, Clemens M, Darb-Esfahani S, Schmitt WD, Dan Costa S, Gerber B, Engels K, Nekljudova V, Loibl S, von Minckwitz G. Nab-paclitaxel versus solvent-based paclitaxel in neoadjuvant chemotherapy for early breast cancer (GeparSepto-GBG 69): a randomised, phase 3 trial. Lancet Oncol 2016; 17: 345-356

[32] Dranitsaris G, Yu B, Wang L, Sun W, Zhou Y, King J, Kaura S, Zhang A, Yuan P. Abraxane ${ }^{\circledR}$ versus Taxol ${ }^{\circledR}$ for patients with advanced breast cancer: A prospective time and motion analysis from a Chinese health care perspective. J Oncol Pharm Pract 2016; 22: 205-211

[33] Ma P, Mumper RJ, Barenholz Y. Anthracycline nano-delivery systems to overcome multiple drug resistance: a comprehensive review. J Control Release 2012; 160: 117-134

[34] Igor VZ, Norbert M, Quet-Fah A. Liposome-encapsulated vincristine, vinblastine and vinorelbine: a comparative study of drug loading and retention. J Control Release 2005; 104: 103-111

[35] Yang SH, Lin CC, Lin ZZ, Tseng YL, Hong RL. A phase 1 pharmacokinetic study of liposomal vinorelbine in patients with advanced solid tumor. Invest New Drugs 2012; 30: 282-289

[36] Wang Y, Dou L, He H, Zhang Y, Shen Q. Multifunctional nanoparticles as nanocarrier for vincristine sulphate delivery to overcome tumor multidrug resistance. Mol Pharm 2014; 11: 885-894

[37] Patankar N, Waterhouse D. Nano-particulate drug delivery systems for camptothecins. Cancer Ther 2012; 8: 90-104

[38] Cuong NV, Hsieh MF, Huang CM. Recent development in nano-sized dosage forms of plant alkaloid camptothecin-derived drugs. Recent Pat Anticancer Drug Discov 2009; 24: 254-261

[39] Isacchi B, Arrigucci S, la Marca G, Bergonzi MC, Vannucchi MG, Novelli A, Bilia AR. Conventional and long-circulating liposomes of artemisinin: preparation, characterization, and pharmacokinetic profile in mice. J Liposome Res 2011; 21: 237-244

[40] Isacchi B, Bergonzi MC, Grazioso M, Righeschi C, Pietretti A, Severini C, Bilia AR. Artemisinin and artemisinin plus curcumin liposomal formulations: enhanced antimalarial efficacy against Plasmodium berghei-infected mice. Eur J Pharm Biopharm 2012; 80: 528-534

[41] Righeschi C, Coronnello M, Mastrantoni A, Isacchi B, Bergonzi MC, Mini E, Bilia AR. Strategy to provide a useful solution to effective delivery of dihydroartemisinin: development, characterization and in vitro studies of liposomal formulations. Colloids Surf B Biointerfaces 2014; 116 : $121-127$

[42] Leto I, Coronnello M, Righeschi C, Bergonzi MC, Mini E, Bilia AR. Enhanced efficacy of artemisinin loaded in transferrin-conjugated liposomes versus stealth liposomes against HCT-8 colon cancer cells. ChemMedChem 2016; 11: 1745-1751

[43] Efferth T, Romero MR, Bilia AR, Osman AG, ElSohly M, Wink M, Bauer R Khan I, Bergonzi MC, Marin JJG. Expanding the therapeutic spectrum of artemisinin: activity against infectious diseases beyond malaria and novel pharmaceutical developments. World J Tradit Chin Med 2016; 2: 1-23

[44] Yallapu MM, Nagesh PK, Jaggi M, Chauhan SC. Therapeutic applications of curcumin nanoformulations. AAPS J 2015; 17: 1341-1356

[45] Song Q, Wang X, Hu Q, Huang M, Yao L, Qi H, Qiu Y, Jiang X, Chen J, Chen $\mathrm{H}$, Gao X. Cellular internalization pathway and transcellular transport of pegylated polyester nanoparticles in Caco-2 cells. Int J Pharm 2013; 445: 58-68

[46] Kundu P, Mohanty C, Sahoo SK. Antiglioma activity of curcumin-loaded lipid nanoparticles and its enhanced bioavailability in brain tissue for effective glioblastoma therapy. Acta Biomater 2012; 8: 2670-2687

[47] Kakkar V, Singh S, Singla D, Kaur IP. Exploring solid lipid nanoparticles to enhance the oral bioavailability of curcumin. Mol Nutr Food Res 2011; 55: 495-503

[48] Righeschi C, Bergonzi MC, Isacchi B, Bazzicalupi C, Gratteri P, Bilia AR. Enhanced curcumin permeability by SLN formulation: The PAMPA approach. LWT-Food Sci Technol 2016; 66: 475-483 
[49] Bergonzi MC, Hamdouch R, Mazzacuva F, Isacchi B, Bilia AR. Optimization, characterization and in vitro evaluation of curcumin microemulsions. LWT-Food Sci Technol 2014; 59: 148-155

[50] Tahmasebi Mirgani M, Isacchi B, Sadeghizadeh M, Marra F, Bilia AR, Mowla SJ, Najafi F, Babaei E. Dendrosomal curcumin nanoformulation downregulates pluripotency genes via miR-145 activation in U87MG glioblastoma cells. Int J Nanomedicine 2014; 9: 403-417

[51] Bhawana I, Basniwal RK, Buttar HS, Jain VK, Jain N. Curcumin nanoparticles: preparation, characterization, and antimicrobial study. J Agric Food Chem 2011; 59: 2056-2061

[52] Rahman M, Beg S, Verma A, Al Abbasi FA, Anwar F, Saini S, Akhter S, Kumar V. Phytoconstituents as pharmacotherapeutics in rheumatoid arthritis: challenges and scope of nano/submicromedicine in its effective delivery. J Pharm Pharmacol 2017; 69: 1-14

[53] Wenzel E, Somoza V. Metabolism and bioavailability of trans-resveratrol. Mol Nutr Food Res 2005; 49: 472-481

[54] Shao J, Li X, Lu X, Jiang C, Hu Y, Li Q, You Y, Fu Z. Enhanced growth inhibition effect of resveratrol incorporated into biodegradable nanoparticles against glioma cells is mediated by the induction of intracellular reactive oxygen species levels. Colloids Surf B Biointerfaces 2009; 72: 40-47

[55] Sanna V, Siddiqui IA, Sechi M, Mukhtar H. Resveratrol loaded nanoparticles based on poly(epsiloncaprolactone) and poly(D,L-lactic-co-glycolic acid)-poly(ethylene glycol) blend for prostate cancer treatment. Mol Pharm 2013; 10: 3871-3881

[56] Wang XX, Li YB, Yao HJ, Ju RJ, Zhang Y, Li RJ, Yu Y, Zhang L, Lu WL. The use of mitochondrial targeting resveratrol liposomes modified with a dequalinium polyethylene glycol distearoylphosphatidyl ethanolamine conjugate to induce apoptosis in resistant lung cancer cells. Biomaterials 2011; 32: 5673-5687

[57] Caddeo C, Teskac K, Sinico C, Kristl ]. Effect of resveratrol incorporated in liposomes on proliferation and UVB protection of cells. Int J Pharm 2008; 363: 183-191

[58] Figueiró F, Bernardi A, Frozza RL, Terroso T, Zanotto-Filho A, Jandrey EH, Moreira JC, Salbego CG, Edelweiss MI, Pohlmann AR, Guterres SS, Battastini AM. Resveratrol-loaded lipid-core nanocapsules treatment reduces in vitro and in vivo glioma growth. J Biomed Nanotechnol 2013; 9: 516-526

[59] Coimbra M, Isacchi B, van Bloois L, Torano JS, Ket A, Wu X, Broere F, Metselaar JM, Rijcken C], Storm G, Bilia AR, Schiffelers RM. Improving solubility and chemical stability of natural compounds for medicinal use by incorporation into liposomes. Int J Pharm 2011; 416: 433-442

[60] Bonaccini L, Karioti K, Bergonzi MC, Bilia AR. Effects of Salvia miltiorrhiza on CNS neuronal injury and degeneration: a plausible complementary role of tanshinones and depsides. Planta Med 2015; 81: 1003-1016

[61] Isacchi B, Fabbri V, Galeotti N, Bergonzi MC, Karioti A, Ghelardini C, Vannucchi MG, Bilia AR. Salvianolic acid B and its liposomal formulations: anti-hyperalgesic activity in the treatment of neuropathic pain. Eur J Pharm Sci 2011; 44: 552-558
[62] Wang X, Deng L, Cai L, Zhang X, Zheng H, Deng C, Duan X, Zhao X, Wei $Y$, Chen L. Preparation, characterization, pharmacokinetics, and bioactivity of honokiol-in-hydroxypropyl- $\beta$-cyclodextrin-in-liposome. J Pharm Sci 2011; 100: 3357-3364

[63] Zhang Y, Chen T, Yuan P, Tian R, Hu W, Tang Y, jia Y, Zhang L. Encapsulation of honokiol into self-assembled pectin nanoparticles for drug delivery to HepG2 cells. Carbohydr Polym 2015; 133: 31-38

[64] Fang F, Gong C, Qian Z, Zhang X, Gou M, You C, Zhou L, Liu J, Zhang Y, Guo G, Gu Y, Luo F, Chen L, Zhao X, Wei Y. Honokiol nanoparticles in thermosensitive hydrogel: therapeutic effects on malignant pleural effusion. ACS Nano 2009; 3: 4080-4088

[65] Wang XH, Cai LL, Zhang XY, Deng LY, Zheng H, Deng CY, Wen JL, Zhao X Wei YQ, Chen LJ. Improved solubility and pharmacokinetics of PEGylated liposomal honokiol and human plasma protein binding ability of honokiol. Int J Pharm 2011; 410: 169-174

[66] Bonifácio BV, Silva PB, Ramos MA, Negri KM, Bauab TM, Chorilli M. Nanotechnology-based drug delivery systems and herbal medicines: a review. Int J Nanomedicine 2014; 9: 1-15

[67] Gunasekaran T, Haile T, Nigusse T, Dhanaraju MD. Nanotechnology: an effective tool for enhancing bioavailability and bioactivity of phytomedicine. Asian Pac J Trop Biomed 2014; 4: S1-S7

[68] Alexander A, Ajazuddin, Patel RJ, Saraf S, Saraf S. Recent expansion of pharmaceutical nanotechnologies and targeting strategies in the field of phytopharmaceuticals for the delivery of herbal extracts and bioactives. J Control Release 2016; 241: 110-124

[69] Javed S, Kohli K, Ali M. Reassessing bioavailability of silymarin. Altern Med Rev 2011; 16: 239-249

[70] Yang KY, Hwang du H, Yousaf AM, Kim DW, Shin Y], Bae ON, Kim YI, Kim JO, Yong CS, Choi HG. Silymarin-loaded solid nanoparticles provide excellent hepatic protection: physicochemical characterization and in vivo evaluation. Int J Nanomedicine 2013; 8: 3333-3343

[71] Yen FL, Wu TH, Lin LT, Cham TM, Lin CC. Nanoparticles formulation of Cuscuta chinensis prevents acetaminophen-induced hepatotoxicity in rats. Food Chem Toxicol 2008; 46: 1771-1777

[72] Valenti D, De Logu A, Loy G, Sinico C, Bonsignore L, Cottiglia F, Garau D, Fadda AM. Liposome-incorporated Santolina insularis essential oil: preparation, characterization and in vitro antiviral activity. J Liposome Res 2001; 11: 73-90

[73] Sinico C, De Logu A, Lai F, Valenti D, Manconi M, Loy G, Bonsignore L, Fadda AM. Liposomal incorporation of Artemisia arborescens L. essential oil and antiviral activity. Eur J Pharm Biopharm 2005; 59: 161-168

[74] Lai F, Sinico C, De Logu A, Zaru M, Müller RH, Fadda AM. SLN as a topical delivery system for Artemisia arborescens essential oil: in vitro antiviral activity and skin permeation study. Int J Nanomedicine 2007; 2: 419-425

[75] Shi F, Zhao JH, Liu Y, Wang Z, Zhang YT, Feng NP. Preparation and characterization of solid lipid nanoparticles loaded with frankincense and myrrh oil. Int J Nanomedicine 2012; 7: 2033-2043 